Aus der Chirurgischen Abteilung des St. Hedwigskrankenhauses in Berlin. (Direktor: Prof. Dr. Rotter.)

\title{
Ueber Wirbelfrakturen.)
}

\section{Von Dr. O. Croce.}

M. H.! Ich will Ihnen über zwei Fälle aus dem Röntgengebiete berichten, die uns lehren, wie notwendig es ist, alle Unfälle - mögen sie auch noch so gering erscheinen, in denen aber der objektive Befund im Widerspruche steht mit den subjektiven Beschwerden des Patienten - zu röntgographieren. um so festzustellen, ob nicht doch irgendwo eine Schädigung sitzt, die man mit anderen Hilfsmitteln nicht feststellen kann: namentlich sollte man dies tun, wenn schwer zugängliche Gebiete betroffen sind, vor allem bei Wirbelsäulenverletzung. Und wie sehr man einen Patienten und nach dessen Tode seine Angehörigen schädigen kann, wenn eine Sicherung der Diagnose durch Röntgogramm unterbleibt, zeigt uns in drastischer Weise der Fall Stempel,2) in welchem laut ärztlichen Gutachtens eine Halswirbeldistorsion angenommen und der nach vier Jahren an Lähmungen infolge Rückenmarksleiden erfolgte Tod als nicht im Zusammenhang mit dem Unfall stehend angesehen wurde. Auf Klage der Angehörigen wurde dann Stempel zum Gutachten aufgefordert, der einen $\mathrm{Zu}$ sammenhang als möglich annahm und die Exhumierung der Leiche dreiviertel Jahr nach dem Tode beantragte, da eine Röntgenaufnahme unterblieben war. Dem Antrag wurde nachgekommen, und die Sektion ergab verschiedene Frakturen am zweiten, sowie Kompressionsfraktur des fünften, sechsten. siebenten Halswirbels. Somit konnte der Tod als Unfallfolge anerkannt werden.

Aehnlich lagen die Verhältnisse auch in unserem Falle, in dem $1^{1 /} / 2$ Jahr eine Dienstmagd auf die verschiedensten Verlegenheitsdiagnosen, wie Rheumatismus, Hysterie und schlieflich Simulation, behandelt wurde. Erst jetzt, da sie Invalidität beantragt hatte und Herr Prof. Rotter sein Gutachten abgeben sollte, konnte, nachdem ich auf Veranlassung des Herrn Prof. Rotter ein Röntgogramm angefertigt hatte, die Ursache der Beschwerden der Patientin in einem isolierten Bruch des vierten Halswirbelkörpers gefunden werden. Der Verlauf des Falles ist in Kürze folgender:

Patientin, 28 Jahre alt, Dienstmagd, stürzte von einem vollgeladenen Heuwagen herab, war kurze Zeit besinnungslos, doch bald darauf ohne größere Beschwerden. Am zweiten Tage stellten sich stärkere Schmerzen im Nacken, namentlich bei Beugung des Kopfes, sowie Schluckschmerz ein. Da nach acht Tagen keine Besserung eintrat, auch der Kopf nach rechts geneigt gehalten wurde, schaffte man sie in ein Krankenhaus, wo sie sieben Wochen lag. Der $\mathrm{Zu}$ stand besserte sich nicht, ohne sich jedoch zu verschlimmern; bis heute bestehen dieselben Beschwerden. Lähmungen oder Sensibilitätsstörungen sind nicht vorhanden.

Röntgogramm-Demonstration (Fig. 1 und 2, Seite 417): Etwas schräger Bruch durch den vierten Halswirbelkörper, Senkung der rechten Bruchhälfte nach unten vorn; zugleich erscheint die rechte Gelenkfläche des Wirbels zusammengedrückt, wodurch ein $\mathrm{Zu}$ sammensinken der Wirbelsäule nach rechts entsteht, während̆ an der linken Seite die Wirbel sich weiter als normal voneinander entfernt haben.

Daß es sich demnach außer diesen Knochenverletzungen um eine schwere Schädigung der nervösen Elemente, des

1) Vortrag, gehalten in der Freien Vereinigung der Chirurgen Berlins am 12. Dezember 1904. - 2) W. Ste m pel, Monatschrift fúr Unfallheikunde 1904, No. 7 
Fig. 1.

Fig. 2.

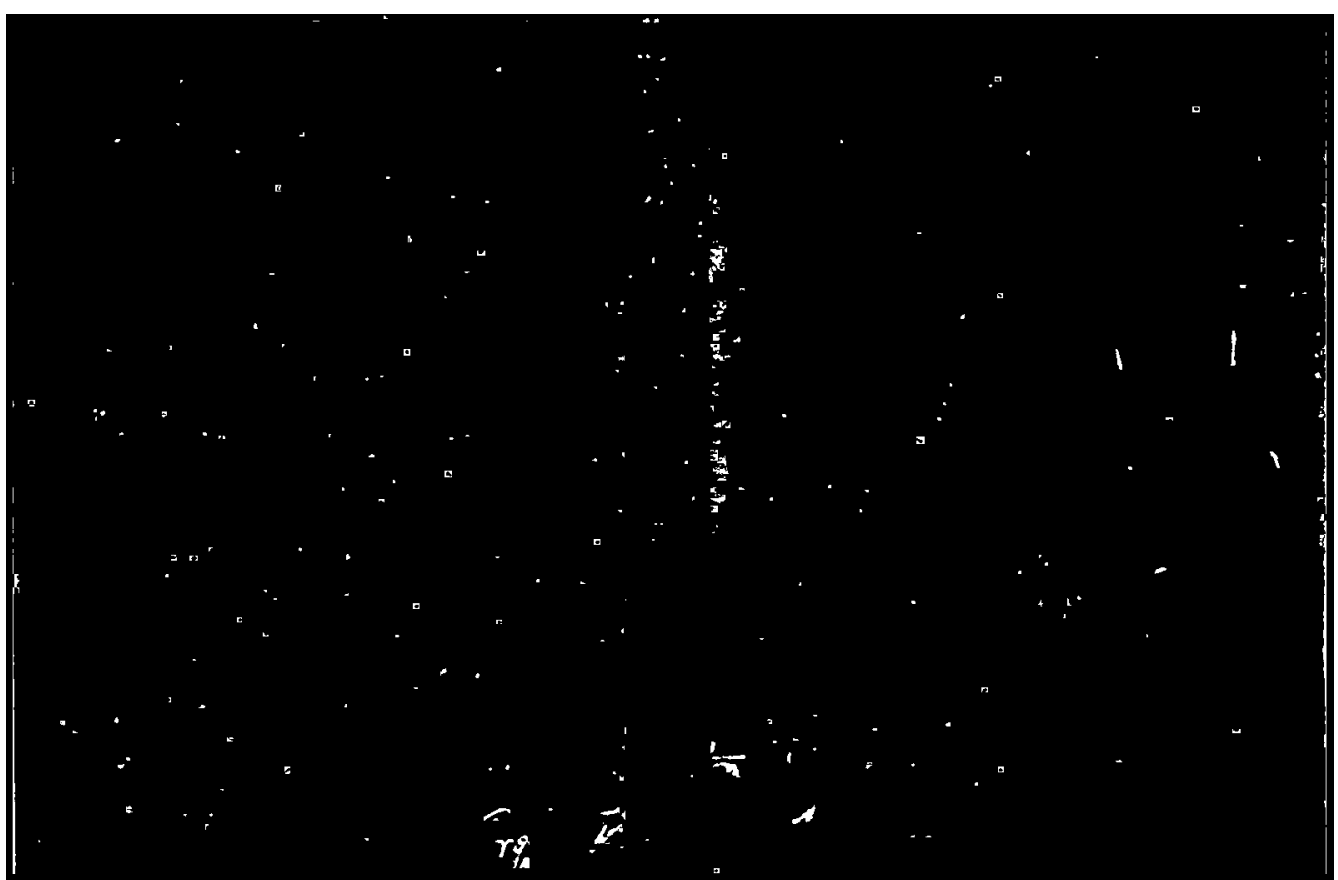

meinem Erstaunen Femur und Becken normal, dagegen im letzten Lendenwirbel eine Fraktur in seinem hinteren Bogen. Das linke Bruchstiick hatte sich iiber das rechte verschoben. (Demonstration les Röntgogramms, Fig. 3.)

Bei dieser Art des Traumas hätte wohl niemand an eine Frak: tur eines Wirbels gedacht Innd demnach auch nicht diagnostiziert, wenn nicht wiederım ein ausgiebiges Nachsuchen durch Röntgoglamme nach der Ursache der Schmerzen die Quelle aufgedeckt hätte.

Es folgt also, meine Herren, aus diesen beiden Fällen, daß man auch bei geringfügigen Traumen, wenn man eine Erklärung fül ${ }^{*}$ die Erscheinungen nicht findet, stets ein Röntgogramm machen und, falls eine Aufnahme noch nicht Klarheit schafft, eine zweite, dritte anschließen soll, namentlich bei Wirbelverletzungen. da sonst of noch eine oder die andere Verletzung übersehen werden wird, auch eine exakte richtige Diagnose nicht zu stellen ist. Im gleichen Sinne spricht sich auch Ludloff

Rückenmarks sowohl als auch der in den Foramina intervertebralia und um sie herum liegenden Teile des Nervenapparates handelt, ist außer allem Zweifel. Das erklürt die Beschwerden der Patientin, sowie die Schiefhaltung des Kopfes vollkommen. Auch ist bezüglich der Prognose diese als zweifelhaft zu stellen, da sich in unserem Falle, wie in dem Falle Stempels, erst nach längerer Zeit ein Rückenmarksleiden einstellen kann. Einen ebensolchen Fall berichtet noch Bernstein ${ }^{1}$, in welchem auch erst am 70. Tage Lähmungserscheinungen und am 101. Tage der Tod eintrat.

Der zweite Fall, den ich Ihnen, meine Herren, kulz berichten möchte, ist noch insofern interessanter, als es sich um ein verbältnismäßig geringfügiges Trauma handelte und niemand anfänglich die Schwere der Verletzung ahnen konnte.

Der Patient, $26 \mathrm{Jahr}$ alt, hagerer, großer, kräftig gebanter Mann, bekam einen Schlag mit einer Wagendeichsel seitlich gegen den linken Trochanter, ohne dabei zu Falle zu kommen.

Bei der Aufnahıne, die bald nach dem Lnfall stattfand, hatte Patient ein ziemlich großes Hämatom um den linken Trochanter. Er konnte das linke Bein nur unter großen Schmerzen und in ganz geringem Maße heben; passiv gelang es leicht, das Bein zu bewegen, ohne Krepitation zu füblen. Patient hatte dabei nur Schmerz am Troehanter. Als auf Bettruhe, Eisblase etc. keine Besserung in den nächsten Tagen eintrat, obwohl das Hamatom fast geschwunden war, dagegen sich neben Parasthesien lancinierende Schmerzen im

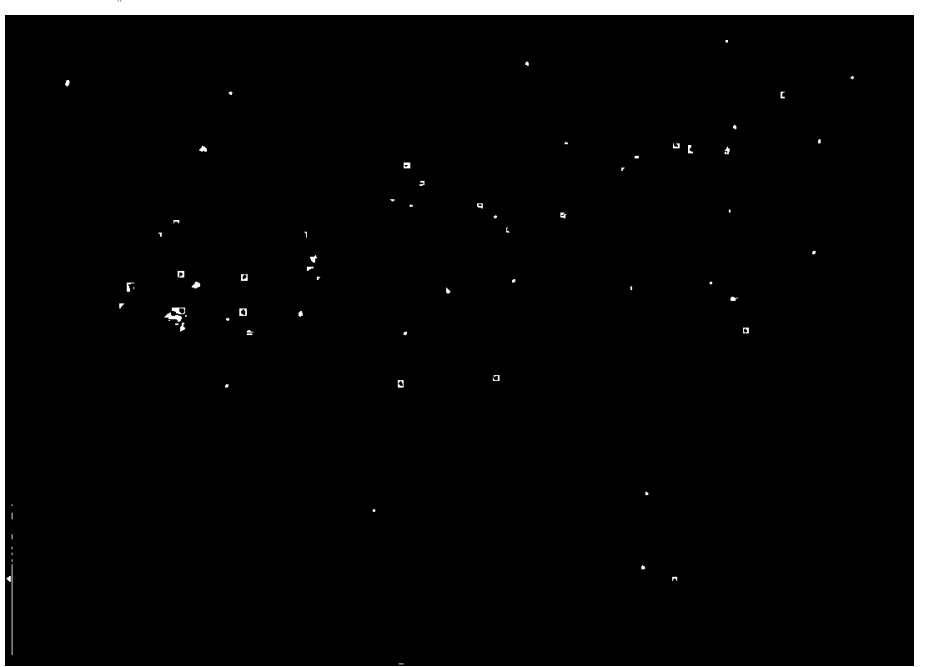

linken Bein wie Kreuzschmerz einstellten. machte ich Röntgen aufnahmen, vom Femur anfangend bis zum Krellybein. und fand zi

\footnotetext{
1) Bernste in, Deutsche Zeitschrift für Chirurgie 1903, Bd. 70.
}

\section{aus. Welcher über 20 Aufnahn des sechsten Halswirbels genau beschreiben konnte.}

